\title{
The effect of feature saliency on haptic subitizing
}

\author{
Myrthe A. Plaisier • Martijn van't Woud • \\ Astrid M. L. Kappers
}

Received: 24 August 2010/Accepted: 27 November 2010/Published online: 25 December 2010

(C) The Author(s) 2010. This article is published with open access at Springerlink.com

\begin{abstract}
Subitizing' refers to fast and error-free numerosity judgment for small $(<4)$ sets of items. For larger sets, the slower process of 'counting' is used. Counting has a serial character, whereas subitizing is believed to have a parallel character. While subitizing was initially found in vision, it has been shown to exist in touch as well. In vision, it has been demonstrated that adding distractor items to a set of target items influences numerosity judgment of the target items. Subitizing was in this case only possible if the distractor item is highly salient among the targets. In the present study, we investigated the effect of adding a distractor item on haptic judgement of a set of target items. To this end, we asked subjects to judge the number of spheres grasped in their hand. Either a cube or an ellipsoid could be added to the set. A cube among spheres has been shown to be highly salient, while an ellipsoid among spheres is not. Our results show that adding a distractor item led to an increase in the response time slopes regardless of the distractor shape. Subitizing was, however, only possible in the case of a salient distractor. This is in agreement with results from vision.
\end{abstract}

Keywords Subitizing - Haptic perception . Feature saliency $\cdot$ Numerosity judgment

M. A. Plaisier $(\square)$ · M. v. Woud · A. M. L. Kappers

Helmholtz Institute, Universiteit Utrecht,

Padualaan 8, $3584 \mathrm{CH}$ Utrecht, The Netherlands

e-mail: m.plaisier@fbw.vu.nl

Present Address:

M. A. Plaisier

Faculty of Human Movement Sciences, VU University,

Van der Boechorststraat 9, 1081 BT Amsterdam,

The Netherlands
From vision it is known that humans can judge the number of items (e.g. dots displayed on a screen) fast and error-free up to about four items (e.g. Atkinson et al. 1976; Mandler and Shebo 1982; Trick and Pylyshyn 1993, 1994; Trick 2008). For larger sets of items, response times and error rates increase rapidly. This second process is generally assumed to be a serial process known as counting, where each item is added to the running total subsequently. For small sets of items, it has been proposed that a faster process known as 'subitizing' is used (Kaufman et al. 1949). It is not yet clear what kind of process subitizing actually is and how it is different from counting. One of the difficulties in distinguishing counting and subitizing is that subitizing can be used in combination with counting for large sets of items. In this case, small subsets of items are subitized and added to the running total (Van Oeffelen and Vos 1982).

Recently, it has been shown that there are striking similarities between visual and haptic numerosity judgement. Subitizing has been shown to exist when subjects had to judge the number of fingers that were stimulated (Riggs et al. 2006), but also when subjects were asked to judge the number of objects grasped in the hand (Plaisier et al. 2009a). When the objects were distributed over both hands, response times could be significantly reduced indicating that, like in vision, subitizing can be used in combination with counting (Plaisier et al. 2010). Also, the shape or size of the objects did not influence numerosity judgement (Plaisier et al. in press). This is again also the case in vision (Trick and Pylyshyn 1994). Finally, relative differences between subsequent numerosities in the set (e.g. the relative difference between 1 and 2 is much larger than between 5 and 6) could not explain a transition from subitizing to counting for either vision or haptics (Plaisier et al. 2010). 
Although similar behavior has been reported for visual and haptic numerosity judgement, the values of the response time slopes are different. Generally, these slopes are larger in haptics than in vision. Visual subitizing and counting slopes are found to be around 0.05 and $0.3 \mathrm{~s} / \mathrm{item}$, respectively (e.g. Akin and Chase 1978; Oyama et al. 1981; Trick and Pylyshyn 1993; Plaisier et al. 2010), while the haptic subitizing slope is generally around 0.2 s/items and the counting slopes range from 0.8 to $1.2 \mathrm{~s} /$ item (Riggs et al. 2006; Plaisier et al. in press; Plaisier et al. 2009a, 2010). The reason why response time slopes are larger in haptics than in vision is not completely clear. It could be that haptic information processing is slower due to noise. It could, however, also be due to the way haptic information is extracted. Haptics usually requires exploratory hand and finger movements that possibly introduce extra processing time. This seems the most likely cause for the variation in counting slopes found in haptics. For counting, generally, all items are visited sequentially involving much more hand and finger movements than subitizing for which the items just need to be grasped in the hand and no further exploration is needed. Nonetheless, in search tasks, the response time slopes are also generally found to be larger than in vision.

From studies into visual search, it is known that some visual features can be detected in parallel, while for others, serial search is necessary (Treisman and Gelade 1980; Treisman and Gormican 1988; Wolfe et al. 1989). In search studies, typically a target item (e.g. a red dot) is presented among a varying number of distractor items (e.g. green dots). The task is to determine as fast as possible whether the target is present. The slope of the response times as a function of the total number of items is a measure for the efficiency at which the search task was performed. Small slopes indicate parallel search, while large slopes indicate serial search. There is a whole range of possible slope values (Wolfe 1998). It is also possible to add distractor items to a set of target items that need to be enumerated. Trick and Pylyshyn (1993) showed that subitizing was still possible if the distractor and target items differ in a feature that can be detected in parallel. More explicitly, they showed that subitizing was possible for determining the number of O's among X's, but not for determining the number of O's among Q's. This led to the conclusion that when a target-distractor combination normally yields serial search, then subitizing is not possible in the presence of such distractors.

Also in the haptic domain search, paradigms have been used to investigate saliency of features like roughness, shape, material properties or temperature differences (Lederman and Klatzky 1997; Overvliet et al. 2008; Plaisier et al. 2008a; Plaisier and Kappers 2010). For threedimensional shapes that could be grasped in the hand, it was found that edges are a highly salient shape feature (Plaisier et al. 2009b). For instance, a cube among spheres could be detected using serial search, while an ellipsoid among spheres was detected through serial search. In the present study, we investigated the effect of adding a distractor item on subitizing of a set of targets. Based on the similarities between visual and haptic numerosity judgement, we would expect similar results to vision. Therefore, we hypothesize that subitizing is possible for a set of spheres when the added distractor is a cube, but not when this distractor is an ellipsoid.

\section{Methods}

\section{Participants}

Ten paid undergraduate students (mean age $21 \pm 2$ years (SD)) participated in the experiment. Three subjects were left-handed according to Coren's test Coren (1993), and all others were right-handed. They were treated in accordance with the local guidelines and signed an informed consent form. They were naive as to the purpose of the experiments, and none of the subjects reported any known hand deficits.

\section{Stimuli and apparatus}

The stimuli consisted of varying numbers of brass shapes, which were suspended from flexible wires. The presented shapes were cubes (edge length $1.5 \mathrm{~cm}$ ), spheres (radius $0.93 \mathrm{~cm}$ ), and ellipsoids (radii: $1.22,0.81$ and $0.81 \mathrm{~cm}$ ). To eliminate weight cues, these dimensions were chosen such that the volume, and thus the weight of the shapes, was constant. The different shapes are shown in Fig. 1a.

A custom-built device was used to measure response times. Time measurement was started when the subject touched the stimulus activating the touch-sensitive contact of the device. The measurement was terminated by a vocal response registered with a headset microphone (for details about this set-up see Plaisier et al. 2008b).

\section{Procedure}

Before the experiment was started, the subjects were informed of which shapes were the target items and which shapes were distractor items. They were instructed to respond the correct number of target items as fast as possible. The subjects wore a blindfold and earplugs to avoid any sound cues. Prior to a trial, they placed their dominant hand with the palm upwards in a holder. Sets of shapes were suspended from flexible wires above the hand, and subjects were instructed to reach upwards to grasp all items simultaneously. This is shown in Fig. 1b. There were no 


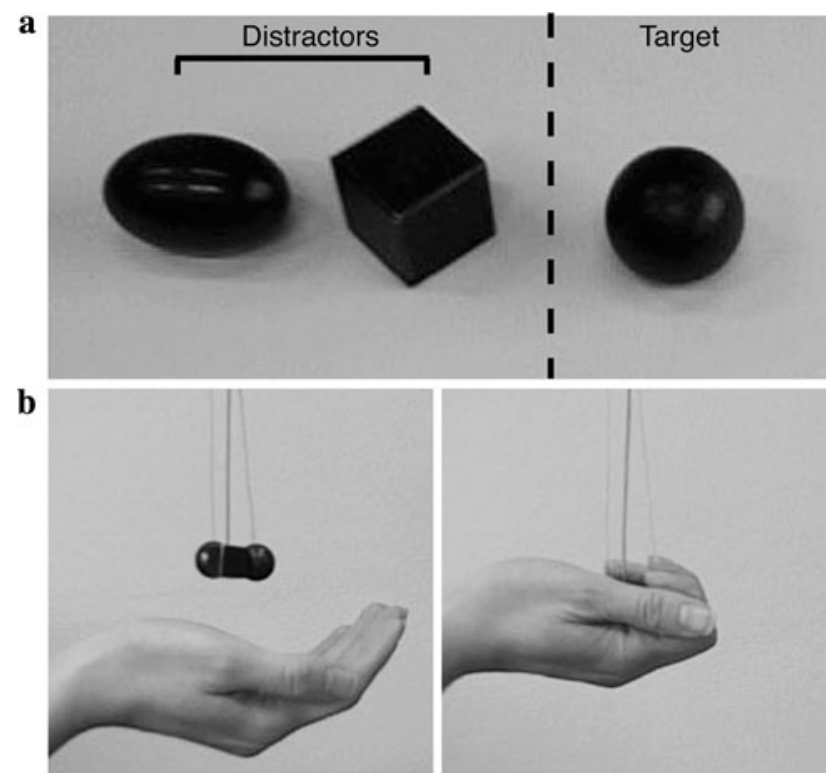

Fig. 1 Stimuli and set-up. a Shapes that were used in the experiment. The targets were always spheres, while the distractor was either a cube or an ellipsoid. b Picture of a subject grasping the stimuli

restrictions on exploratory hand and finger movements. After grasping all items, they were allowed to release items from their hand during the trial. It was emphasized that they should only release items from their hand if this seemed the most efficient strategy. The experimenter scored whether an item was released from the hand during each trial. Subjects were instructed to respond the correct number of target items as fast as possible. They received feedback from the experimenter on whether the answer was correct. Error trials were repeated at the end of a block of trials, and only correct responses were included in the analysis.

The two conditions were performed in separate blocks of trials on separate days in counter-balanced order. In one condition, a cube was added as distractor item, and in the other condition, an ellipsoid was used as distractor item. In both cases, spheres were the target items, and the number of spheres had to be judged. In half of the trials, a distractor item was present, and the distractor-present and distractorabsent trials were randomly interleaved. There could be 1-4 target items to cover the whole subitizing range. The subitizing range in haptics was never reported to exceed 3 items. We measured up to 4 items, but did not include this numerosity in the analysis to exclude possible end-effects. Each numerosity was repeated ten times with and without a distractor in the set leading to a total of $(10 \times 4 \times 2=) 80$ trials per block. To each block of trials also 15 catch trials were added. In the catch trials, the presented set of items could contain up to 4 distractor items with the restriction that the total number of items in the set did not exceed 6 . This was done to prevent subjects from noticing there was always only one distractor item, but these trials were not included in the analysis. A similar procedure was used by Trick and Pylyshyn (1993). Prior to each block of trials, subjects performed at least 20 training trials, and trials were continued until 10 subsequent responses were correct. It was never necessary to exceed 30 training trials.

Analysis

Because subjects were instructed to respond as fast as possible, but also correct the error rate should be low in all conditions. Therefore, the error rates are given as an indication that subjects could perform the task correctly. Only the response times and item release rates were used for further analysis. Incorrectly answered trials were excluded from the analysis. Weighted linear regression was performed on the response times averaged over subjects as well as on the single subjects' data. Note that this does not necessarily yield the same outcome. The quality of fit to the data averaged over subjects will be better, but it is also important to show that the same trend is present for each subject individually. For this reason, the results from both procedures are reported. The largest numerosity was not included in the regression analysis of the reported slopes. The last numerosity in the presented range of numerosities normally deviates from linearity and is therefore often excluded from the regression analysis (e.g Trick and Pylyshyn 1993; Watson et al. 2007; Trick 2008). The results from the present study were, however, not significantly different when the largest numerosity was included in the analysis. Furthermore, repeated measures ANOVA was performed on the single-subject regression slopes as well as on the item release rate.

To determine the effect on the slope values, we compare the response times slopes to the values of response time slopes found in two previous studies performed without any distractors (Plaisier et al. 2009a, 2010). These studies were performed using the same spheres as used in the present study, and the same response time measuring device was used. Comparison between these studies seems therefore justified. In the two previous studies, sets of up to 7 or 12 items were used, and a regression of a bi-linear function was used to determine the subitizing and counting slope values as well as the transition point between the two regimes.

\section{Results}

The error rates were overall low. For the distractor-absent trials, error rates were $0.5 \%$ in the cube condition and $0.75 \%$ in the ellipsoid condition. In the distractor-present trials, the error rates were 3 and $4.25 \%$, respectively. Response times averaged over subjects as a function of the 
number of items with a cube as distractor are shown in Fig. 2a, and response times with an ellipsoid as distractor are shown in Fig. 2b. The response times for the distractorabsent trials are shown in black and those for the distractorpresent trials are shown in gray. The solid lines represent weighted linear regression to averaged response times. This yielded in the condition with a cube as distractor, a distractor-absent slope of $0.2 \mathrm{~s} /$ item and a distractor-present slope of $0.6 \mathrm{~s} /$ item. For the condition with the ellipsoid as distractor, the distractor-absent slope was found to be $1.1 \mathrm{~s} /$ items, and the distractor-present slope was $1.6 \mathrm{~s} /$ item.

Linear regression was also performed on the singlesubject data. The values from the single-subject slopes averaged again over subjects and the standard deviation between subjects are reported here. This yielded in the cube condition a distractor-absent slope of $0.2 \pm 0.1 \mathrm{~s} /$ item $\left(R^{2}=0.8 \pm 0.1\right)$ and a distractor-present slope of $0.6 \pm$ 0.2 s/item $\left(R^{2}=0.9 \pm 0.1\right)$. For the ellipsoid condition, this yielded a distractor-absent slope of $1.1 \pm 0.3$ s/item $\left(R^{2}=0.99 \pm 0.02\right)$ and a distractor-present slope of $1.6 \pm 0.6$ s/item $\left(R^{2}=0.98 \pm 0.02\right)$. A $2 \times 2$ (distractor shape $\times$ distractor presence) repeated measures ANOVA was performed on the single-subject slopes. There was an effect of distractor shape $(F(1,9)=47, p<0.001)$ and of distractor presence $(F(1,9)=22, p=0.001)$. There was no interaction effect between distractor shape and distractor presence.

The percentage of trials in which items were released from the hand is shown in Fig. 3. A $2 \times 2$ (distractor shape $\times$ distractor presence) repeated measures ANOVA was performed on the item release rates. This showed an effect of distractor shape $(F(1,9)=34, p<0.001)$ as well as distractor presence $(F(1,9)=107, p<0.001)$ and an interaction effect $(F(1,9)=7, p=0.025)$.

\section{Discussion}

Our results show that subitizing slopes increased when a distractor was added, regardless of the saliency of the distractor. When the distractor was a cube, the numerosity

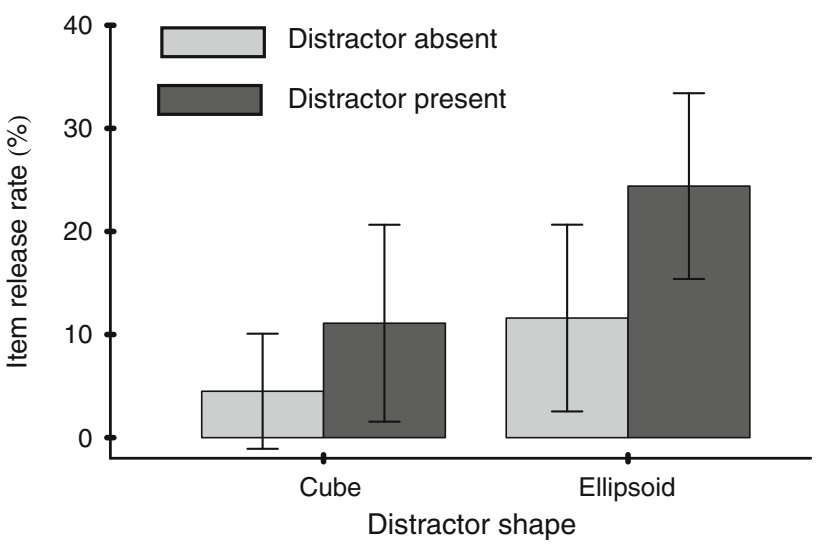

Fig. 3 Item release rate collapsed over all numerosities and averaged over subjects for distractor-present and distractor-absent trials in both conditions. The error bars indicate the between-subjects standard deviation

judgment slope increased from 0.2 to $0.6 \mathrm{~s}$ /item. When the distractor was an ellipsoid, slopes increased from 1.1 to 1.6 s/item. In two previous studies into haptic numerosity judgment, we measured the subitizing and counting slopes when the items were spheres (Plaisier et al. 2009a, 2010). Slope values were found to be 0.17 and $0.2 \mathrm{~s} /$ items in the subitizing regime and $0.8 \mathrm{~s} /$ item and $1.2 \mathrm{~s} /$ item in the counting regime. The slopes found in the present study for the ellipsoid condition have a value comparable to counting slopes. This means that in the ellipsoid condition, there was no subitizing. The slope values for the cube condition were, however, clearly below counting slope values. The distractor-absent slope was in this case in the same range as subitizing slopes that were found previously. The distractor-present slope was in between subitizing and counting slope values. This shows that adding a distractor increases numerosity judgement slopes, but subitizing was still possible in the cube condition.

The distractor-absent slope in the cube condition was comparable to previously found subitizing slope values, but the distractor-absent slope in the ellipsoid condition was clearly in the range of generally found counting slopes. The stimulus was, however, the same in both cases. Since a
Fig. 2 Response times averaged over subjects for the distractor-present and distractor-absent trials. a Condition with a cube as distractor and $\mathbf{b}$ condition with an ellipsoid as distractor. The solid lines indicate linear regression to the response times, and the error bars indicate the between subjects standard deviation
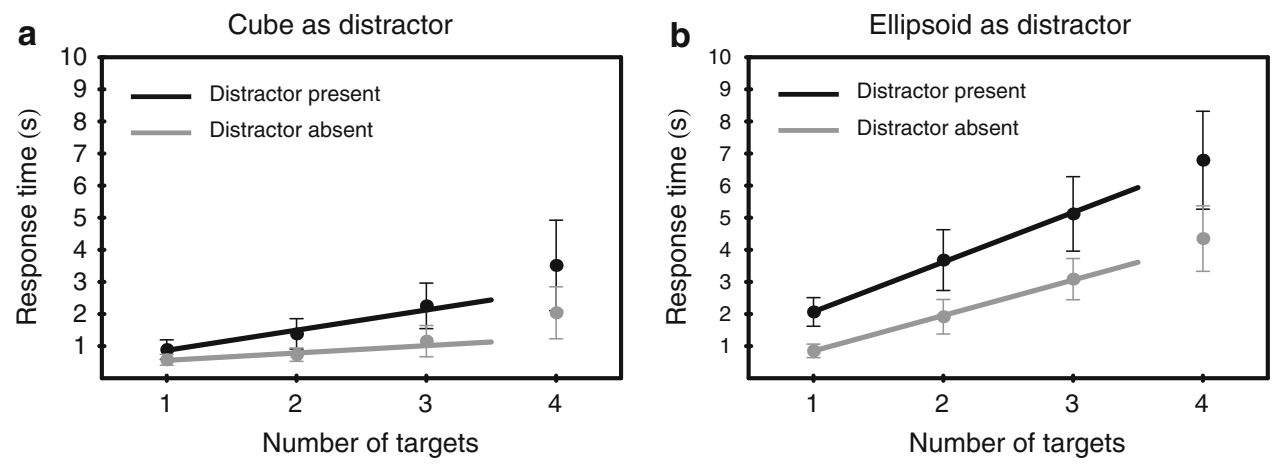
cube among spheres is highly salient subjects knew in the cube condition immediately after grasping, the set whether there were distractors present. Once they knew there were no distractors, they subitized the whole set. When cubes were detected, they should be ignored while judging the numerosity, and this resulted in an increase in the slope. An ellipsoid among spheres, on the other hand, is not salient, and serial search is needed to find it. This is also indicated by the larger item release rate in the ellipsoid condition than in the cube condition. Releasing items from the hand is normally an indication of serial processing (Plaisier et al. 2009b). Therefore, subjects visited each item subsequently regardless of whether a distractor was present in the ellipsoid condition. Still, in this case, the slope was larger when a distractor was present. So, although items were processed in a serial fashion, the presence of a distractor increased the response time per item. Note that this difference in slope cannot be caused by the fact that in distractor-present trials, the total number of items was larger than in the distractor-absent case. This causes a larger offset of the response times, but does not affect the slopes.

Also in vision, it was found that the slope for small sets of items increased when a distractor was added, even if this was a highly salient distractor (Trick and Pylyshyn 1993). Similar to our results, also the slope for distractor-absent trials differed depending on the type of distractor used. When a non-salient distractor was used, subitizing was possible for the distractor-absent trials, but the slope was larger than when a salient distractor was used. The effects reported here for haptic subitizing in the presence of a distractor are therefore comparable to those found in vision. The effect of adding a single distractor to the set was in the haptic case, however, more pronounced than in the visual case.

Our results suggest a modality-independent process underlying subitizing. Trick and Pylyshyn (1993) concluded from the fact that subitizing was only possible when the targets 'pop-out' from among the distractors that there is a pre-attentive stage of item individuation in subitizing. They proposed that there are 'fingers of instantiation' (FINST's) that can be used to point to individual items in the visual field (see Pylyshyn 2001 for a review). These 'fingers' do not need to be attributed to item properties, such as color, shape or even position. Although this visual theory might be extended to include the haptic modality, an alternative explanation which is not modality specific is that of a capacity limited short term memory. It has been shown that humans can hold a limit number of chunks of information in their working memory. Such a capacity limit would result in the same upper limit for subitizing in vision and touch which generally seems to be true (see Cowan 2001 for an overview). A third explanation of subitizing does not involve assuming any capacity limits.
Peterson and Simon (2000) computationally showed that when varying numbers of dots are placed on a twodimensional grid, there are many more recurrent patterns for small numerosities $(<4)$ than for larger numerosities. They then showed that their neural network displayed subitizing-like behavior after a learning period. Such a pattern recognition explanation is, however, less likely in the haptic case as the objects were not fixed in space. The grasped objects could be freely moved in the hand. Therefore, a capacity limit in short term memory would be the best candidate for a modality transcending explanation for the occurrence of subitizing.

Nor in the present study, nor in the study by (Trick and Pylyshyn 1993) was the subitizing slope unaffected by adding distractors. This suggests impairment of the individuation of the targets from the distractors. It is clearly not caused by impairment of the subitizing process as the subitizing slopes were at their normal values in distractorabsent trials when pop-out targets were used. If first the targets were pre-attentively selected and then put into memory chunks, we would not expect the subitizing slope to be affected at all for pop-out targets. The same holds for the pattern repetition explanation. Therefore, it seems that either distractors cannot be completely ignored during subitizing or the total set size is determined first and the distractors are subtracted afterwards to arrive at the number of targets. Either way, salient distractors impair subitizing in both vision and haptics although they do not prevent subitizing like non-salient distractors do.

Acknowledgments This work was financed by the European Union as part of a CP-Large-Scale integrating project The Hand Embodied (consortium agreement: ICT-248587-THE)

Open Access This article is distributed under the terms of the Creative Commons Attribution Noncommercial License which permits any noncommercial use, distribution, and reproduction in any medium, provided the original author(s) and source are credited.

\section{References}

Akin O, Chase W (1978) Quantification of three-dimensional structures. J Exp Psychol Hum Percept Perform 4:397-410

Atkinson J, Campbell FW, Francis MR (1976) The magic number $4 \pm 0$ : a new look at visual numerosity judgments. Perception 5:327-334

Coren S (1993) The left-hander syndrome: the causes and consequences of left-handedness. Vintage Books, New York

Cowan N (2001) Metatheory of storage capacity limits. Behav Brain Sci 24(01):154-176

Kaufman E, Lord M, Reese T, Volkmann J (1949) The discrimination of visual number. Am J Psychol 62:498-525

Lederman SJ, Klatzky RL (1997) Relative availability of surface and object properties during early haptic processing. J Exp Psychol Hum Percept Perform 23:1680-1707 
Mandler G, Shebo BJ (1982) Subitizing: an analysis of its component processes. J Exp Psychol Gen 111:1-22

Overvliet KE, Smeets JBJ, Brenner E (2008) The use of proprioception and tactile information in haptic search. Acta Psychol 129:83-90

Oyama T, Kikuchi T, Ichihara S (1981) Span of attention, backward masking, and reaction time. Percept Psychophys 29:106-112

Peterson S, Simon T (2000) Computational evidence for the subitizing phenomenon as an emergent property of the human cognitive architecture. Cogn Sci 24(1):93-122

Plaisier MA, Kappers AML (2010) Cold objects pop out! In: Kappers AML, Erp JBF, Bergmann Tiest WM, Helm FCT (eds) Haptics: generating and perceiving tangible sensations, vol 6192 of Lecture Notes on Computer Science. Springer, Berlin, pp 219-224

Plaisier MA, Bergmann Tiest WM, Kappers AML (2008a) Haptic pop-out in a hand sweep. Acta Psychol 128:368-377

Plaisier MA, Bergmann Tiest WM, Kappers AML (2008b) Haptic search for spheres and cubes. In: Ferre $M$ (eds) Haptics: perception, devices and scenarios, vol 5024 of Lecture Notes on Computer Science. Springer, Berlin, pp 275-282

Plaisier MA, Bergmann Tiest WM, Kappers AML (2009a) One, two, three, many-subitizing in active touch. Acta Psychol 131:163-170

Plaisier MA, Bergmann TWM, Kappers AML (2009b) Salient features in three-dimensional haptic shape perception. Attent Percept Psychophys 71(2):421-430

Plaisier MA, Bergmann Tiest WM, Kappers AML (2010) Range dependent processing of visual numerosity: similarities across vision and haptics. Exp Brain Res 204:525-537

Plaisier MA, Bergmann Tiest WM, Kappers AML (in press) Haptic object individuation. IEEE Trans Haptics
Plaisier MA, Bergmann Tiest WM, Kappers AML (2010) Grabbing subitizing with both hands: bimanual number processing. Exp Brain Res 202:507-512

Pylyshyn ZW (2001) Visual indexes, preconceptual objects, and situated vision. Cogn Tech Work 80:127-158

Riggs KJ, Ferrand L, Lancelin D, Fryziel L, Dumur G, Simpson A (2006) Subitizing in tactile perception. Psychol Sci 17:271-272

Treisman A, Gelade G (1980) A feature-integration theory of attention. Cogn Psychol 12:97-136

Treisman A, Gormican S (1988) Feature analysis in early vision: evidence from search asymmetries. Psychol Rev 95:15-48

Trick LM (2008) More than superstition: differential effects of featural heterogeneity and change on subitizing and counting. Percept Psychophys 70:743-760

Trick LM, Pylyshyn ZW (1993) What enumeration studies can show us about spatial attention: evidence for limited capacity preattentive processing. J Exp Psychol Hum Percept Perform 19:331-351

Trick LM, Pylyshyn ZW (1994) Why are small and large numbers enumerated differently? A limited-capacity preattentive stage in vision. Psychol Rev 101:80-102

Van Oeffelen MP, Vos PG (1982) Configurational effects on the enumeration of dots: counting by groups. Memory Cogn 10:396-404

Watson DG, Maylor EA, Bruce LAM (2007) The role of eye movements in subitizing and counting. J Exp Psychol Hum Percept Perform 33:1389-1399

Wolfe JM (1998) What can 1 million trials tell us about visual search? Psychol Sci 9:33-39

Wolfe JM, Cave KR, Franzel SL (1989) Guided search: an alternative to the feature integration model for visual search. J Exp Psychol Hum Percept Perform 15:419-433 